\title{
On the induction of new fuzzy relations, new fuzzy operators and their aggregation
}

\author{
Neus Carmona, Jorge Elorza, Jordi Recasens, Jean Bragard
}

\begin{abstract}
In this paper we generate fuzzy relations and fuzzy operators using different kind of generators and we study the relationship between them. Firstly, we introduce a new fuzzy preorder induced by a fuzzy operator. We generalize this preorder to a fuzzy relation generated by two fuzzy operators and we analyze its properties. Secondly, we introduce and explore two ways of inducing a fuzzy operator, one from a fuzzy operator and a fuzzy relation and the other one from two fuzzy operators. The first one is an extension of the well-known fuzzy operator induced by a fuzzy relation through Zadeh's compositional rule. Finally, we aggregate these operators through the quasi-arithmetic mean associated to a continuous Archimedean t-norm in order to compare the operator obtained by aggregating the generators with the operator obtained by the aggregation of the generated ones.
\end{abstract}

\section{Introduction}

Fuzzy relations and fuzzy consequence operators are main concepts in fuzzy logic. The fuzzy relation induced by a fuzzy operator and the fuzzy operator induced by a fuzzy relation through Zadeh's compositional rule are notions that have been extensively explored (see for instance $[?, ?, ?, ?, ?, ?])$.

In Section ?? we recall the main definitions and results that will be used throughout the paper.

Neus Carmona, Jorge Elorza, Jean Bragard

Departamento de Física y Matemática Aplicada, Facultad de Ciencias, Universidad de Navarra, P.O. Box 31008 Pamplona, Spain

e-mail: ncarmona@alumni.unav.es, e-mail: jelorza@unav.es,e-mail: jbragard@unav.es

Jordi Recasens

ETS Arquitectura del Vallès, Department of Mathematics and Computer Sciences, c/ Pere Serra 1-15, P.O. Box 08190 Sant Cugat del Vallès, Spain

e-mail: j.recasens@upc.edu 
In Section ?? we introduce a fuzzy preorder $R_{c}^{c}$ induced by a fuzzy operator $c$ such that collects the information of $c$ over all the fuzzy subsets of the universal set. Recall that the classical relation induced by a fuzzy operator only considers the information over the singletons. We generalize this preorder to a fuzzy relation $R_{f}^{g}$ induced by two fuzzy operators $f$ and $g$ and study its properties. From a logical point of view, $R_{f}^{g}$ stablishes a crossed relation between the consequences of $g$ and the consequences of $f$.

In Section ?? we define two new operators $C_{R}^{g}$ and $C_{f}^{g}$. The first one is induced by a fuzzy relation and a fuzzy operator and the second one is induced by two fuzzy operators. We explore the properties that are transmitted from the generators. In particular, we show for wich cases the properties of a fuzzy consequence operator (inclusion, monotony and idempotence) and the coherence property hold.

In Section ?? we aggregate fuzzy operators using the quasi-arithmetic mean associated to a continuous Archimedean t-norm. We study the particular case of $C_{R}^{g}$ and $C_{f}^{g}$ and the difference between aggregating the generators or the generated operators.

Finally, in Section ?? we present the conclusions.

\section{Preliminaries}

Let $\langle L, \wedge, \vee, *, \rightarrow, 0,1\rangle$ be a complete commutative residuated lattice in the sense of Bělohlávek [?]. That is, a complete lattice $\langle L, \wedge, \vee, 0,1\rangle$, where 0 denotes the least element and 1 denotes the greatest one, such that $(L, *)$ is a commutative monoid i.e. $*$ is associative, commutative and with neutral element 1 , and the operations $*$ and $\rightarrow$ satisfy the adjointness property:

$$
x * y \leq z \quad \Leftrightarrow \quad y \leq x \rightarrow z
$$

where $\leq$ denotes the lattice ordering.

Let us recall in Propositions ?? and ?? the following properties of commutative residuated lattices (residuated lattices for short) [?] that will be used in the paper.

Proposition 1. Each residuated lattice $\langle L, \wedge, \vee, *, \rightarrow, 0,1\rangle$ satisfies the following conditions for all $x, y, z \in X$ :
1. $x \rightarrow x=1$
4. $x * 0=0$
2. $1 \rightarrow x=x$
5. $x *(x \rightarrow y) \leq y$
3. $x \leq y \Leftrightarrow x \rightarrow y=1$
6. $(x \rightarrow y) *(y \rightarrow z) \leq(x \rightarrow z)$

Proposition 2. Let $\langle L, \wedge, \vee, *, \rightarrow, 0,1\rangle$ be a residuated lattice. The following conditions hold for each index set I whenever both sides of the (in)equality exist. In the first case, if the left hand side makes sense, so does the right one. For all $x, y_{i} \in L$ with $i \in I$,

1. $x * \bigvee_{i \in I} y_{i}=\bigvee_{i \in I}\left(x * y_{i}\right)$ 
2. $x * \bigwedge_{i \in I} y_{i} \leq \bigwedge_{i \in I}\left(x * y_{i}\right)$

The frame for our work will be the complete commutative residuated lattice $\langle[0,1], \wedge, \vee, *, \rightarrow, 0,1\rangle$ where $\wedge$ and $\vee$ are the usual infimum and supremum, $*$ is a left continuous t-norm and $\rightarrow$ is the residuum of $*$ defined for $\forall a, b \in X$ as $a \rightarrow b=\sup \{\gamma \in[0,1] \mid a * \gamma \leq b\}$. Recall that a t-norm is monotone in both arguments and the residuum is antitone in the first argument and monotone in the second one.

In this paper, $X$ will be a non-empty classical universal set, $[0,1]^{X}$ will be the set of all fuzzy subsets of $X, \Gamma^{\prime}$ will denote the set of all fuzzy relations defined on $X$ and $\Omega^{\prime}$ the set of fuzzy operators defined from $[0,1]^{X}$ to $[0,1]^{X}$.

Definition 1. (Fuzzy Consequence Operator) A fuzzy operator $C \in \Omega^{\prime}$ is called a fuzzy consequence operator when it satisfies for all $\mu, v \in[0,1]^{X}$ :

(C1) Inclusion $\mu \subseteq C(\mu)$

(C2) Monotony $\mu \subseteq v \Rightarrow C(\mu) \subseteq C(v)$

(C3) Idempotence $C(C(\mu))=C(\mu)$

The inclusion of fuzzy subsets is given by the puntual order, i.e. $\mu \subseteq v$ if and only if $\mu(x) \leq v(x)$ for all $x \in X$.

Definition 2. (Coherent Fuzzy Operator) Let $C \in \Omega^{\prime}$ be a fuzzy operator in $\Omega^{\prime}$. We say that $C$ is coherent if it satisfies for all $x, a \in X$ and $\mu \in[0,1]^{X}$

$$
\mu(a) * C(\{a\})(x)) \leq C(\mu)(x)
$$

Let us look back on some properties of fuzzy relations. A fuzzy relation on $X$ is said to be:

(R) Reflexive if $R(x, x)=1 \quad \forall x \in X$

(S) Symmetric if $R(x, y)=R(y, x) \quad \forall x, y \in X$

(T) $*$-Transitive if $R(x, y) * R(y, z) \leq R(x, z) \quad \forall x, y, z \in X$

A fuzzy relation satisfying $(\mathrm{R})$ and $(\mathrm{T})$ is called a fuzzy preoder. If it also satisfies (S), then it is called a fuzzy similarity or indistinguishability operator. Given $R$ and $S$ fuzzy relations, we say that $R \leq S$ if and only if $R(x, y) \leq S(x, y)$ for all $x, y \in X$.

For a given fuzzy relation $R$, a fuzzy subset $\mu$ of $X$ is called $*$-compatible with $R$ if $\mu(x) * R(x, y) \leq \mu(y)$ for all $x, y \in X$. From its logical implications, these sets are also called true-sets or closed under modus ponens. This notion gets special interest when $R$ is a preorder [?]. When $R$ is not only a preorder but also an indistinguishability operator, these sets are called extensional sets and the set of all these subsets has very interesting properties [?].

Every fuzzy operator induces a fuzzy relation in a very natural way and every fuzzy relation also induces a fuzzy operator using Zadeh's compositional product: 
Definition 3. Let $C$ be a fuzzy operator in $\Omega^{\prime}$. The fuzzy relation induced by $C$ is given by

$$
R_{C}(x, y)=C(\{x\})(y)
$$

where $\{x\}$ denotes the singleton $x$.

Definition 4. Let $R \in \Gamma^{\prime}$ be a fuzzy relation on X. The fuzzy operator induced by $R$ through Zadeh's compositional rule is defined by

$$
C_{R}^{*}(\mu)(x)=\sup _{w \in X}\{\mu(w) * R(w, x)\}
$$

These concepts are strongly connected and they have been extensively explored in several contexts (see for instance $[?, ?, ?, ?, ?, ?])$.

\section{Relation Induced by Fuzzy Operators}

Notice that the relation induced by (??) only takes into account the behaviour of $C$ over the singletons and not over more general fuzzy subsets. In order to include this information, we define a new fuzzy relation induced by a fuzzy operator in a different way.

Definition 5. Let $c$ be a fuzzy operator in $\Omega^{\prime}$. The fuzzy relation $R_{c}^{c}$ induced by $c$ is given by

$$
R_{c}^{c}(x, y)=\inf _{\mu \in[0,1]^{X}}\{c(\mu)(x) \rightarrow c(\mu)(y)\}
$$

It is easy to see that this relation is a fuzzy preorder on $X$ since it is the infimum of a family of preorders. From a logical point of view, the crisp interpretation of this relation would be

$$
x \leq y \text { (or related to } \mathrm{y} \text { ) } \Leftrightarrow \underset{ }{\forall A} \subseteq X \text {, if } x \text { is a consequence of } A \text { then } y \text { is also a }
$$

Notice that if $c$ is an inclusive operator, then $R_{c}^{c} \leq R_{c}$. In fact, for all $x, y \in X$ we have $R_{c}^{c}(x, y)=\inf _{\mu \in[0,1]^{x}}\{c(\mu)(x) \rightarrow c(\mu)(y)\} \leq c(\{x\})(x) \rightarrow c(\{x\})(y)=$ $R_{c}(x, y)$.

In Definition ?? we generalize the previous definition to the fuzzy relation $R_{f}^{g}$ induced by two fuzzy operators $f$ and $g . R_{f}^{g}$ is a crossed relation whose logical interpretation in the crisp case would be the following

$\mathrm{x}$ is related to $\mathrm{y} \Leftrightarrow$ whenever $\mathrm{x}$ is a consequence by $g$ of some subset $A$, then $y$ is a consequence of the same subset by $f$.

Definition 6. Let $f$ and $g$ be fuzzy operators in $\Omega^{\prime}$. The fuzzy relation $R_{f}^{g}$ induced by $f$ and $g$ is defined by 


$$
R_{f}^{g}(x, y)=\inf _{\mu \in[0,1]^{X}}\{g(\mu)(x) \rightarrow f(\mu)(y)\}
$$

$g$ and $f$ will be called the upper and lower generators of $R_{f}^{g}$ respectively.

In Propositions ?? and ?? we study the reflexive and $*$-trasitive properties of $R_{f}^{g}$.

Proposition 3. Let $f$ and $g$ be fuzzy operators in $\Omega^{\prime}$. Then, $R_{f}^{g}$ is reflexive if and only if $g \leq f$, i.e. $g(\mu)(x) \leq f(\mu)(x)$ for all $\mu \in[0,1]^{X}$ and $x \in X$.

Proof.

$$
\begin{aligned}
R_{f}^{g} \text { is reflexive } & \Leftrightarrow R_{f}^{g}(x, x)=1 \quad \forall x \in X \\
& \Leftrightarrow \inf _{\mu \in[0,1]^{X}}\{g(\mu)(x) \rightarrow f(\mu)(x)\}=1 \quad \forall x \in X \\
& \Leftrightarrow g(\mu)(x) \rightarrow f(\mu)(x)=1 \quad \forall \mu \in[0,1]^{X}, \quad \forall x \in X \\
& \Leftrightarrow g(\mu)(x) \leq f(\mu)(x) \quad \forall \mu \in[0,1]^{X}, \quad \forall x \in X \quad \Leftrightarrow \quad g \leq f
\end{aligned}
$$

Proposition 4. Let $f, g \in \Omega^{\prime}$ be fuzzy operators with $f \leq g$. Then, the induced fuzzy relation $R_{f}^{g}$ is $*$-transitive.

Proof.

$$
\begin{aligned}
R_{f}^{g}(x, y) * R_{f}^{g}(y, z) & =\inf _{\mu \in[0,1]^{X}}\{g(\mu)(x) \rightarrow f(\mu)(y)\} * \inf _{\mu \in[0,1]^{X}}\{g(\mu)(y) \rightarrow f(\mu)(z)\} \\
& \leq \inf _{\mu \in[0,1]^{X}}\{(g(\mu)(x) \rightarrow f(\mu)(y)) *(g(\mu)(y) \rightarrow f(\mu)(z))\} \\
& \leq \inf _{\mu \in[0,1]^{X}}\{(g(\mu)(x) \rightarrow g(\mu)(y)) *(g(\mu)(y) \rightarrow f(\mu)(z))\} \\
& \leq \inf _{\mu \in[0,1]^{X}}\{g(\mu)(x) \rightarrow f(\mu)(z)\}=R_{f}^{g}(x, z)
\end{aligned}
$$

\section{Inducing Fuzzy Operators from Different Generators}

In this section we introduce two new operators $C_{R}^{g}$ and $C_{f}^{g}$. Their construction is based on Zadeh's compositional rule in a very similar way to the construction given by (??). In this case, it involves either a fuzzy relation $R$ and a fuzzy operator $g$ or two fuzzy operators $f, g$ (generators).

Definition 7. Let $g \in \Omega^{\prime}$ be a fuzzy operator and let $R \in \Gamma^{\prime}$ be a fuzzy relation on $\mathrm{X}$. We define the operator $C_{R}^{g}$ induced by $g$ and $R$ as

$$
C_{R}^{g}(\mu)(x)=\sup _{w \in X}\{g(\mu)(w) * R(w, x)\}
$$

$R$ and $g$ are called the generators of $C_{R}^{g}$. 
Notice that $C_{R}^{*}$ is a particular case of $C_{R}^{g}$. Taking $g=i d$, where $i d$ denotes the identity operator on $[0,1]^{X}$, we obtain $C_{R}^{i d}=C_{R}^{*}$.

Definition 8. Let $g, f \in \Omega^{\prime}$ be fuzzy operators. The operator $C_{f}^{g}$ induced by $g$ and $f$ is defined by

$$
C_{f}^{g}(\mu)(x)=\sup _{w \in X}\{g(\mu)(w) * f(\{w\})(x)\}
$$

$g$ and $f$ will be called the upper and lower generators of $C_{f}^{g}$ respectively.

The following result shows some basic properties of $C_{R}^{g}$ and $C_{f}^{g}$.

Proposition 5. Given $g_{1}, g_{2}, f_{1}, f_{2}$ fuzzy operators and $R_{1}, R_{2}$ fuzzy relations, the following holds

1. If $g_{1} \leq g_{2}$, then $C_{R}^{g_{1}} \leq C_{R}^{g_{2}} \quad \forall R \in \Gamma^{\prime}$

2. If $R_{1} \leq R_{2}$ then $C_{R_{1}}^{g} \leq C_{R_{2}}^{g} \quad \forall g \in \Omega^{\prime}$

3. If $f_{1} \leq f_{2}$ then $C_{f_{1}}^{g} \leq C_{f_{2}}^{g} \quad \forall g \in \Omega^{\prime}$

4. If $g_{1} \leq g_{2}$ then $C_{f}^{g_{1}} \leq C_{f}^{g_{1}} \quad \forall f \in \Omega^{\prime}$

Proof. All implications directly follow from the monotony of $*$. To illustrate it, we will prove ??. For any $\mu \in[0,1]^{X}$ and $x \in X$ we have

$C_{f}^{g_{1}}(\mu)(x)=\sup _{y \in X}\left\{g_{1}(\mu)(y) * f(\{y\})(x)\right\} \leq \sup _{y \in X}\left\{g_{2}(\mu)(y) * f(\{y\})(x)\right\}=C_{f}^{g_{2}}(\mu)(x)$

There exists a close relationship between the operators $C_{f}^{g}$ and $C_{R}^{g}$.

Theorem 1. For every pair $(g, f)$ of fuzzy operators, there exists a fuzzy relation $R$ such that $C_{R}^{g}=C_{f}^{g}$. $R$ is uniquely determined. Conversely, for every pair $(g, R)$ of a fuzzy operator and a fuzzy relation, there exists at least a fuzzy operator $f$ such that $C_{f}^{g}=C_{R}^{g}$.

Proof. To prove the first statement of the theorem, notice that given $(g, f)$ and using the usual definition $R_{f}(x, y)=f(\{x\})(y), C_{f}^{g}$ coincides with $C_{R_{f}}^{g}$. The unicity follows from the construction.

To prove the second statement, notice that for every fuzzy relation $R \in \Gamma^{\prime}$ we can define a fuzzy operator $f_{R}$ as follows:

$$
f_{R}(\mu)(y)= \begin{cases}R(x, y) & \text { if } \mu \text { is the singleton }\{x\} \\ \mu(y) & \text { if } \mu \text { is not a singleton }\end{cases}
$$

Then, for all $\mu \in[0,1]^{X}$ and $x \in X$,

$$
C_{f_{R}}^{g}(\mu)(x)=\sup _{w \in X}\left\{g(\mu)(w) * f_{R}(\{w\})(x)\right\}=\sup _{w \in X}\{g(\mu)(w) * R(w, x)\}=C_{R}^{g}(\mu)(x)
$$


Remark 2 Observe that there are infinite choices for the operator $f_{R}$ since we are only concerned about its effect over the singletons.

Remark 3 From Theorem ?? we can conclude that every property satisfied for $C_{f}^{g}$ for arbitrary $f$ will also be satisfied for $C_{R}^{g}$ for arbitrary $R$. Conversely, every property satisfied for $C_{R}^{g}$ for arbitrary $R$ will also be satisfied for $C_{f}^{g}$ for arbitrary $R$.

Given $f, g$ two operators and $C_{f}^{g}$ the operator that they generate, there is a fuzzy relation $R$ such that $C_{f}^{g}=C_{R}^{g}$ and it is exactly $R_{f}$. Suppose that a property is satisfied for $C_{R}^{g}$ for every $R \in \Gamma^{\prime}$. It will particulary be satisfied for $C_{R_{f}}^{g}$. Hence, it will also be satisfied for $C_{f}^{g}$.

On the other hand, for any relation $R \in \Gamma^{\prime}$ and $g \in \Omega^{\prime}$ there exist an infinite number of operators $f_{R}$ for which $C_{f_{R}}^{g}$ coincides with $C_{R}^{g}$. Every property satisfied for $C_{f}^{g}$ for an arbitrary $f$ will be satisfied for $C_{f_{R}}^{g}$ independently of the $f_{R}$ chosen. Hence, it will also be satisfied for $C_{R}^{g}$.

Let us study which properties of $C_{f}^{g}$ and $C_{R}^{g}$ are transmitted from the generators. Our main interest is to characterize for which generators we obtain fuzzy consequence operators (FCO).

Lemma 4 Let $g \in \Omega^{\prime}$ and $R \in \Gamma^{\prime}$. If $R$ is reflexive, then $C_{R}^{g} \geq g$.

Proof. $C_{R}^{g}(\mu)(x)=\sup _{w \in X}\{g(\mu)(w) * R(w, x)\} \geq g(\mu)(x) * R(x, x)=g(\mu)(x)$

Proposition 6. Let $g \in \Omega^{\prime}$ be an inclusive fuzzy operator and $R \in \Gamma^{\prime}$ a reflexive fuzzy relation. Then, $C_{R}^{g}$ is also an inclusive fuzzy operator.

Proof. From lemma ?? and the inclusion of $g, C_{R}^{g}(\mu)(x) \geq g(\mu)(x) \geq \mu(x)$.

We have an equivalent result for the inclusion of $C_{f}^{g}$.

Proposition 7. Let $g \in \Omega^{\prime}$ be an inclusive fuzzy operator and $f \in \Omega^{\prime}$ a fuzzy operator which is inclusive over the singletons. Then, $C_{f}^{g}$ is also an inclusive fuzzy operator.

Proof. Since $f$ is inclusive over the singletons, the relation $R_{f}(x, y)=f(\{x\})(y)$ is reflexive. From the proof of Theorem ??, we know that $C_{f}^{g}=C_{R_{f}}^{g}$. Then, it follows from the previous proposition that $C_{f}^{g}$ is also inclusive.

Proposition 8. Let $g \in \Omega^{\prime}$ be a monotone fuzzy operator. Then, $C_{R}^{g}$ is also a monotone fuzzy operator for any $R \in \Gamma^{\prime}$.

Proof. Suppose $\mu_{1} \subseteq \mu_{2}$. Then, $g\left(\mu_{1}\right)(x) \leq g\left(\mu_{2}\right)(x)$ for all $x \in X$ and it follows that $C_{R}^{g}\left(\mu_{1}\right)(x)=\sup _{w \in X}\left\{g\left(\mu_{1}\right)(w) * R(w, x)\right\} \leq \sup _{w \in X}\left\{g\left(\mu_{2}\right)(w) * R(w, x)\right\}=C_{R}^{g}\left(\mu_{2}\right)(x)$ 
Remark 5 Notice that Proposition ?? and Remark ?? ensure that if $g$ is a monotone fuzzy operator, then $C_{f}^{g}$ is also monotone for any $f \in \Omega^{\prime}$.

Thus, $C_{f}^{g}$ and $C_{R}^{g}$ inherit the monotony of its upper generator $g$. This is due to the fact that $g$ has an effect over general fuzzy subsets. Notice that neither the lower generator $f$ nor $R$ do. Hence, the monotony of the lower generator $f$ does not imply the monotony of $C_{f}^{g}$ as it is shown in the following simple example.

Example 6 Let $f$ be the identity operator which is trivially monotone. Let $g$ be any operator which is not monotone. Then, $C_{f}^{g}(\mu)(x)=\sup _{y \in X}\{g(\mu)(y) * f(\{y\})(x)\}=$ $\{g(\mu)(x) *\{x\}(x)\}=g(\mu)(x)$. Since $g$ is not monotone, neither is $C_{f}^{g}$.

The idempotence does not follow from the idempotence of the generators as directly as the inclusion or the monotony do. In order to generate a FCO from another FCO, we require an additional property. We need the subsets from the image of the upper generator $g$ to be $*$-compatible with the given relation.

Definition 9. Let $g$ be a fuzzy operator and $R$ a fuzzy relation. We will say that $g$ is $*$-concordant with $R$ if all the subsets from the image of $g$ are $*$-compatible with $\mathrm{R}$.

Theorem 7. Let $R \in \Gamma^{\prime}$ be a reflexive fuzzy relation and let $g \in \Omega^{\prime}$ be a FCO. Suppose that $g$ is $*$-concordant with $R$. Then, the operator $C_{R}^{g}$ induced by $g$ and $R$ is also a FCO.

Proof. Propositions ?? and ?? give us the properties of inclusion and monotony of $C_{R}^{g}$. It only remains to prove the idempotence. To prove the first inclusion notice that, since $g(\mu)$ belongs to $\operatorname{Im}(g)$, it is *-compatible with $R$, so $g(\mu)(y) * R(y, x) \leq$ $g(\mu)(x)$ for all $y, x \in X$. Hence, $\sup _{y \in X}\{g(\mu)(y) * R(y, x)\} \leq g(\mu)(x)$ for all $x \in X$. Using this fact, the monotony and idempotence of $g$ and the monotony of $*$ we get

$$
\begin{aligned}
C_{R}^{g}\left(C_{R}^{g}(\mu)\right)(x) & =\sup _{w \in X}\left\{g\left(C_{R}^{g}(\mu)\right)(w) * R(w, x)\right\} \\
& =\sup _{w \in X}\left\{g\left(\sup _{y \in X}\{g(\mu)(y) * R(y, w)\}\right) * R(w, x)\right\} \\
& \leq \sup _{w \in X}\{g(g(\mu)(w)) * R(w, x)\} \\
& =\sup _{w \in X}\{g(\mu)(w) * R(w, x)\}=C_{R}^{g}(\mu)(x)
\end{aligned}
$$

The other inclusion follows immediately from the inclusion property.

Remark 8 We can state an equivalent result for the operator $C_{f}^{g}$. Let $g$ and $f$ be two fuzzy operators such that $g$ is FCO and $f$ is inclusive over the singletons. If $g$ is *-concordant with $R_{f}(x, y)=f(\{x\})(y)$, then $C_{f}^{g}$ is a FCO.

Let us prove that the coherence property is inherited from the upper generator.

Proposition 9. Let $g \in \Omega^{\prime}$ be a coherent fuzzy operator and $R$ a fuzzy relation in $X$. Then, $C_{R}^{g}$ is also a coherent fuzzy operator. 
Proof. Using property ?? from Proposition ?? we have that $\forall a \in X$ and $\forall \mu \in[0,1]^{X}$,

$$
\begin{aligned}
\mu(a) * C_{R}^{g}(\{a\})(x) & =\mu(a) * \sup _{y \in X}\{g(\{a\})(y) * R(y, x)\} \\
& =\sup _{y \in X}\{\mu(a) * g(\{a\})(y) * R(y, x)\} \\
& =\sup _{y \in X}\{(\mu(a) * g(\{a\})(y)) * R(y, x)\} \\
& \leq \sup _{y \in X}\{g(\mu)(y) * R(y, x)\}=C_{R}^{g}(\mu)(x)
\end{aligned}
$$

where the inequality holds because of the coherence of $g$.

Remark 9 From remark ?? we can state the same about the coherence of $C_{f}^{g}$. That is, if $g$ is a coherent fuzzy operator, then $C_{f}^{g}$ is also a coherent fuzzy operator.

\section{Aggregation of Fuzzy Operators through the Quasi-arithmetic Mean}

In this section, we will assume that $*$ is not only a left-continuous t-norm, but also Archimedean and with an additive generator $t$. Let us recall that a t-norm is Archimedean if for each $x, y \in(0,1)$ there is an $n \in \mathbb{N}$ with $x^{n}=x *{ }^{n} * *<<y$. An additive generator of a t-norm is a strictly decreasing function $t:[0,1] \rightarrow[0, \infty]$, right continuous in 0 , with $t(1)=0$ and satisfying $t(x)+t(y) \in \operatorname{Ran}(t) \cup[t(0), \infty]$ such that

$$
x * y=t^{(-1)}(t(x)+t(y))
$$

where $t^{(-1)}$ denotes the pseudo-inverse of $t$ defined as:

$$
t^{(-1)}(y)=\sup \{x \in[0,1] \mid t(x)>y\}
$$

The left-continuity of a t-norm $*$ with additive generator $t$, implies its continuity and therefore, the continuity of its generator. In this case, the pseudo-inverse becomes the usual inverse of $t$ [?].

Given a continuous Archimedean t-norm $*$ with additive generator $t$, there is a natural way to define the extended quasi-arithmetic mean associated to $*$, $m_{t}: \bigcup_{n \in \mathbb{N}}[0,1]^{n} \longrightarrow[0,1]$ (see [?]):

$$
m_{t}\left(x_{1}, \ldots, x_{n}\right)=t^{-1}\left(\frac{1}{n} \sum_{i=1}^{n} t\left(x_{i}\right)\right)
$$

Given a finite family of fuzzy operators, we can aggregate them using the quasiarithmetic mean associated to $*$ in order to obtain another fuzzy operator. 
Definition 10. (Quasi-arithmetic mean of fuzzy operators) Let $t:[0,1] \rightarrow[0, \infty]$ be an additive generator of a continuous Archimedean t-norm $*$. Let $\left\{g_{1}, . ., g_{n}\right\}$ be a finite family of fuzzy operators. The $n$-ary quasi-arithmetic mean generated by $t$ is the fuzzy operator given by

$$
m_{t}\left(g_{1}, \ldots, g_{n}\right)=t^{-1}\left(\frac{1}{n} \sum_{i=1}^{n} t\left(g_{i}\right)\right)
$$

such that for every fuzzy subset $\mu \in[0,1]^{X}$ and every $x \in X$ is

$$
m_{t}\left(g_{1}, \ldots, g_{n}\right)(\mu)(x)=t^{-1}\left(\frac{1}{n} \sum_{i=1}^{n} t\left(g_{i}(\mu)(x)\right)\right)
$$

The extended quasi-arithmetic mean generated by $t$ is the function $m_{t}: \bigcup_{n \in \mathbb{N}}\left(\Omega^{\prime}\right)^{n} \rightarrow$ $\Omega^{\prime}$ that maps any finite family of $n$ fuzzy operators to their n-ary quasi arithmetic mean.

The quasi-arithmetic mean can be defined more generally [?]. Indeed, it can be defined for any continuous and strictly increasing or strictlty decreasing function $f:[0,1] \longrightarrow[-\infty, \infty]$. In this case, the expression $\infty-\infty$ needs to be defined (it is often considered $-\infty$ ). However, we will focus on the natural case where the generator of $m_{t}$ is the additive generator of the given continuous Archimedean tnorm $*$.

Remark 10 Observe that, if $g_{1}, \ldots, g_{n} \in \Omega^{\prime}$ are fuzzy operators. Then, their arithmetic mean satisfies that

$$
\min \left(g_{1}, \ldots, g_{n}\right) \leq m_{t}\left(g_{1}, \ldots, g_{n}\right) \leq \max \left(g_{1}, \ldots, g_{n}\right)
$$

It is known that the quasi-arithmetic mean $m_{t}$ generated by $t$ is strictly increasing and idempotent (in the sense that $m_{t}(g, \ldots, g)=g$ ) if the generator is continuous and stricly increasing or strictly decreasing [?]. From this fact, the next two propositions follow:

Proposition 10. Let $g_{1}, . ., g_{n} \in \Omega^{\prime}$ be inclusive fuzzy operators. Then, its quasi arithmetic mean is also an inclusive fuzzy operator.

Proposition 11. Let $g_{1}, . ., g_{n} \in \Omega^{\prime}$ be monotone fuzzy operators. Then, its quasi arithmetic mean is also a monotone fuzzy operator.

Remark 11 Observe that the idempotence of the $g_{i}$ is in general not translated into the idempotence of their quasi-arithmetic mean. Consider for example the quasi arithmetic mean of $g_{1}=i d$ and $g_{2}=\frac{1}{2}$ id with the product $t$-norm.

Consider the operators $C_{f}^{g}$ and $C_{R}^{g}$ from the previous section. Given a finite family of fuzzy operators, let us compare two different processes of aggregation through the quasi-arithmetic mean. The first one by aggregating the generators, the second one by aggregating the generated operators. 
Theorem 12. Let $g_{1}, . ., g_{n} \in \Omega^{\prime}$ be fuzzy operators and $t:[0,1] \longrightarrow[0, \infty]$ be an additive generator of the continuous Archimedean $t$-norm $*$. Let $m_{t}$ be the extended quasi-arithmetic mean generated by $t$. Then, for every $f \in \Omega^{\prime}$ and every $R \in \Gamma^{\prime}$

$$
C_{f}^{m_{t}\left(g_{1}, \ldots, g_{n}\right)} \leq m_{t}\left(C_{f}^{g_{1}}, \ldots, C_{f}^{g_{n}}\right) \quad \text { and } \quad C_{R}^{m_{t}\left(g_{1}, \ldots, g_{n}\right)} \leq m_{t}\left(C_{R}^{g_{1}}, \ldots, C_{R}^{g_{n}}\right)
$$

Proof. We prove the first inequality:

$$
\begin{aligned}
& C_{f}^{m_{t}\left(g_{1}, \ldots, g_{n}\right)}(\mu)(x)=\sup _{w \in X}\left\{m_{t}\left(g_{1}, \ldots, g_{n}\right)(\mu)(w) * f(\{w\})(x)\right\} \\
= & \sup _{w \in X}\left\{t^{-1}\left(\frac{\sum_{i=1}^{n} t\left(g_{i}(\mu)(w)\right)}{n}\right) * f(\{w\})(x)\right\} \\
= & \sup _{w \in X}\left\{t^{-1}\left(t\left[t^{-1}\left(\frac{\sum_{i=1}^{n} t\left(g_{i}(\mu)(w)\right)}{n}\right)\right]+t(f(\{w\})(x))\right)\right\} \\
= & \sup _{w \in X}\left\{t^{-1}\left(\frac{t\left(g_{1}(\mu)(w)\right)+\cdots+t\left(g_{n}(\mu)(w)\right)}{n}+t(f(\{w\})(x))\right)\right\} \\
= & \sup _{w \in X}\left\{t^{-1}\left(\frac{t\left(g_{1}(\mu)(w)\right)+\cdots+t\left(g_{n}(\mu)(w)\right)+n \cdot t(f(\{w\})(x))}{n}\right)\right\} \\
= & \sup _{w \in X}\left\{t^{-1}\left(\frac{t\left(g_{1}(\mu)(w)\right)+t(f(\{w\})(x))}{n}+\cdots+\frac{t\left(g_{n}(\mu)(w)\right)+t(f(\{w\})(x))}{n}\right)\right\} \\
= & \sup _{w \in X}\left\{t^{-1}\left(\frac{t\left(g_{1}(\mu)(w) * f(\{w\})(x)\right)}{n}+\cdots+\frac{t\left(g_{n}(\mu)(w) * f(\{w\})(x)\right)}{n}\right)\right\} \\
\leq & t^{-1}\left(\frac{t\left(\sup _{w \in X}\left\{g_{1}(\mu)(w) * f(\{w\})(x)\right\}\right)}{n}+\cdots+\frac{t\left(\sup _{w \in X}\left\{g_{n}(\mu)(w) * f(\{w\})(x)\right\}\right)}{n}\right) \\
= & t^{-1}\left(\frac{1}{n} \sum_{i=1}^{n} t\left(\sup _{w \in X}\left\{g_{i}(\mu)(w) * f(\{w\})(x)\right\}\right)\right)=m_{t}\left(C_{f}^{g_{1}}, \ldots, C_{f}^{g_{n}}\right)(\mu)(x)
\end{aligned}
$$

Finally, we can prove the following Theorem similarly to the previous one:

Theorem 13. Let $f_{1}, \ldots, f_{n} \in \Omega^{\prime}$ be fuzzy operators and $t:[0,1] \longrightarrow[0, \infty]$ be an additive generator of the continuous Archimedean t-norm $*$. Let $m_{t}$ be the extended quasi-arithmetic mean generated by $t$. Then, for every $g \in \Omega^{\prime}$,

$$
C_{m_{t}\left(f_{1}, \ldots, f_{n}\right)}^{g} \leq m_{t}\left(C_{f_{1}}^{g}, \ldots, C_{f_{n}}^{g}\right)
$$

\section{Conclusions}

In this paper we have generated fuzzy relations and fuzzy operators using different kind of generators and we have studied their properties. We have defined a fuzzy relation induced by two operators $f, g$ that uses more information than the behaviour 
of $f$ and $g$ over the singletons. We have proved that this relation is reflexive if and only if $g \leq f$, $*$-transitive when $f \leq g$ and a preorder when $f=g$.

We have defined two fuzzy operators $C_{R}^{g}$ and $C_{f}^{g}$, the first one induced by a fuzzy relation and a fuzzy operator and the second one induced by two fuzzy operators. We have shown that they are equivalent in the following sense: For every $C_{f}^{g}$, there exists $R$ such that $C_{f}^{g}=C_{R}^{g}$. Conversely, for every $C_{R}^{g}$ there exists $f$ such that $C_{R}^{g}=C_{f}^{g}$.

We have defined the $*$-concordance of a fuzzy operator with a fuzzy relation and we have shown that for a FCO $g$ which is $*$-concordant with a reflexive fuzzy relation $R$, the generated $C_{R}^{g}$ is also a FCO. The same is true for $C_{f}^{g}$ if $g$ and the relation $R_{f}$ induced by $f$ in the classical way satisfy the mentioned conditions. We have also shown that the coherence property is directly transmitted from the upper generator.

Finally, we have introduced $C_{f}^{m_{t}\left(g_{i}\right)}$ and $C_{m_{t}\left(f_{i}\right)}^{g}$ by aggregation using the quasiarithmetic mean associated to a continuous Archimedean t-norm. We have shown that for a finite family of fuzzy operators $C_{f}^{m_{t}\left(g_{i}\right)} \leq m_{t}\left(C_{f}^{g_{i}}\right)$ and $C_{m_{t}\left(f_{i}\right)}^{g} \leq m_{t}\left(C_{f_{i}}^{g}\right)$. The same holds for $C_{R}^{g}$, thus $C_{R}^{m_{t}\left(g_{i}\right)} \leq m_{t}\left(C_{R}^{g_{i}}\right)$.

Acknowledgements We ackowledge the partial support of the project FIS2011-28820-C02-02 from the Spanish Government and N.C. acknowledges the financial support of the "Asociación de Amigos de la Universidad de Navarra".

\section{References}

1. Bělohlávek, R.: Fuzzy Relational Systems: Foundations and Principles. Ifsr International Series on Systems Science and Engineering, V. 20, Kluwer Academic/Plenum Publishers, New York (2002)

2. Castro J.L. and Trillas, E.: Tarskis fuzzy consequences. In: Proceedings of the International Fuzzy Engineering Symposium '91, Vol. 1, pp. 70-81 (1991)

3. Castro, J.L., Delgado, M. and Trillas, E.: Inducing implication relations. International Journal of Approximate Reasoning, Vol. 10, Issue 3, pp. 235-250 (1994)

4. Grabisch, M., Marichal, J.L., Mesiar, R. and Pap, E.: Aggregation Functions (Encyclopedia of Mathematics and its Applications). Cambridge University Press (2009)

5. Elorza, J. and Burillo, P.: On the relation of fuzzy preorders and fuzzy consequence operators, International Journal of Uncertainty, Fuzziness and Knowledge-based Systems 7 (3), pp. 219 234 (1999)

6. Elorza, J. and Burillo, P.:Connecting fuzzy preorders, fuzzy consequence operators and fuzzy closure and co-closure systems. Fuzzy Sets and Systems, 139(3), pp. 601-613 (2003)

7. Elorza, J. et al.: On the relation between fuzzy closing morphological operators, fuzzy consequence operators induced by fuzzy preorders and fuzzy closure and co-closure systems. Fuzzy Sets and Systems (2012), http://dx.doi.org/10.1016/j.fss.2012.08.010

8. Klement, E.P. , Mesiar, R. and Pap, E.: Triangular Norms, Trends in logic, Studia logica library, Springer (2000)

9. Recasens, J.: Indistinguishability Operators: Modelling Fuzzy Equalities and Fuzzy Equivalence Relations. Studies in Fuzziness and Soft Computing, V. 260, Springer (2010) 